\title{
dltA overexpression: A strain-independent keystone of daptomycin resistance in methicillin-resistant Staphylococcus aureus
}

\author{
Viviana Cafiso ${ }^{a}$, Taschia Bertuccio ${ }^{a}$, Simona Purrello ${ }^{a}$, Floriana Campanile ${ }^{a}$, \\ Caterina Mammina ${ }^{\mathrm{b}}$, Assunta Sartor ${ }^{\mathrm{c}}$, Annibale Raglio ${ }^{\mathrm{d}}$, Stefania Stefani ${ }^{\mathrm{a}, *}$ \\ a Department of Biomedical Sciences-Microbiology, University of Catania, Via Androne 81, 95124 Catania, Italy \\ b Department of Sciences for Healthy Promotion 'G. D'Alessandro', University of Palermo, Palermo, Italy \\ ${ }^{\text {c } M i c r o b i o l o g y}$ Unit, Santa Maria della Misericordia University Hospital, Udine, Italy \\ d USC Microbiologia e Virologia, AO Ospedali Riuniti, Bergamo, Italy
}

\section{A R T I C L E I N F O}

\section{Article history:}

Received 26 July 2013

Accepted 4 October 2013

\section{Keywords:}

MRSA

Daptomycin

Glycopeptides

Real-time qPCR

Sequencing

\begin{abstract}
A B S T R A C T
The mechanisms leading to reduced susceptibility to daptomycin (DAP) are multifactorial and have not been fully elucidated. We analysed, by sequencing and expression studies, the role of the major molecular targets (cell-envelope charge genes, dltA, mprF, cls2; cell-wall turnover and autolysis genes, sce $D$, $a t l$ involved in the emergence of DAP resistance in three series of isogenic clinical methicillin-resistant Staphylococcus aureus (MRSA) in which DAP resistance emerged after a heterogeneous glycopeptideintermediate S. aureus (hGISA) step under teicoplanin and DAP therapy. All of the isolates had different genotypes and were $\delta$-haemolysin negative, reflecting a strain proclivity to acquire DAP/glycopeptide non-susceptibility under antibiotic pressure. DAP exposure led to the emergence of DAP resistance after an hGISA step probably in parallel with the timing of the two antimicrobial administrations and, in two of three cases, in conditions of DAP underdosage. Real-time qPCR data revealed that all DAP-resistant (DAP$\mathrm{R}$ ) isolates had dltA overexpression, whereas $m p r F$ upregulation was found only in DAP-R strains with the S295L and T345I amino acid substitutions. Strains that were heteroresistant to DAP did not possess DAP-R-like characteristics. DAP-R strains presented high cls2 expression and no known cls2 mutations, and moreover exhibited sceD and atl upregulation. In conclusion, these findings highlight that dltA overexpression is the common pathway of resistance among genotypically different series of isolates and may represent the keystone of DAP resistance in MRSA, leading to electrostatic repulsion and, indirectly, to a reduction of autolysin activity. $m p r F$ mutations related to increased transcription may play a role in this complex phenomenon.
\end{abstract}

(c) 2013 Elsevier B.V. and the International Society of Chemotherapy. All rights reserved.

\section{Introduction}

Daptomycin (DAP) has been increasingly used in the treatment of various types of infections caused by methicillin-resistant Staphylococcus aureus (MRSA) [1]. However, since 2005 cases of DAP-resistant (DAP-R) isolates have been described in the literature $[2,3]$. Although the incidence remains low, non-susceptibility to DAP appears to be the result of single or combined strainrelated and/or infection-related events. Strain-related causes of DAP resistance ${ }^{1}$ are thought to be due to accumulation of multiple mutations in different targets, affecting different cell wall and membrane pathways [4], whilst infection-related causes can be due to: (i) potential exposure of the micro-organisms

\footnotetext{
* Corresponding author. Tel.: +39 095250 4714/311 352; fax: +39 0952504733. E-mail addresses: stefanis@unict.it, simona.purrello@unict.it (S. Stefani). 1 In this study, although the official terminology is 'daptomycin nonsusceptibility', the term 'daptomycin resistance' was used for ease of understanding.
}

to subinhibitory concentrations of the drug owing to large variations in serum peak and trough levels at the currently recommended doses [5]; (ii) prior exposure to other antimicrobial agents, particularly vancomycin (VAN) [6,7]; (iii) a high bacterial inoculum and prolonged antibiotic exposure and/or biofilm-related infections, such as infectious endocarditis or bone infections [8]; and (iv) exposure to host-derived cationic peptides [9].

The emergence of S. aureus with diminished DAP susceptibility during glycopeptide [VAN or teicoplanin (TEC)] therapy represents a challenge for the medical community, as VAN treatment may account for loss of DAP susceptibility $[2,7]$.

The European Committee on Antimicrobial Susceptibility Testing (EUCAST) [10] and the Clinical and Laboratory Standards Institute (CLSI) [11] define an S. aureus strain as DAP-R or DAP non-susceptible at a minimum inhibitory concentration (MIC) cutoff value of $>1 \mathrm{mg} / \mathrm{L}$. Strains with a heterogeneous phenotype, i.e. strains with subpopulations growing at antibiotic concentrations above the MIC, have also been described [12]. 
The phenotypic and molecular features of DAP-R S. aureus, commonly related to glycopeptide reduced susceptibility, can be grouped into: (i) changes in cell-wall arrangement and turnover [13]; (ii) changes in membrane composition, structure and membrane potential [14]; and (iii) modifications in sensitivity to autolysis and permeabilisation [14]. Several genetic loci have been implicated in S. aureus non-susceptibility to DAP and glycopeptides, such as ditABCD (alanylation of wall teichoic acids), $\operatorname{mprF}$ (lysinylation of phosphatidylglycerol), sceD, atl and lytM (autolysins), two-component regulatory systems such as WalRK and GraRS, and the agr (accessory gene regulator) locus $[15,16]$. In particular, mprF encodes a bifunctional membrane protein mediating both the lysinylation of phosphatidylglycerol and its translocation to the outer leaflet of the membrane. The ditABCD operon controls the alanylation of wall teichoic acids in response to antimicrobial challenge; its pathway is linked to cationic antimicrobial peptide resistance in S. aureus, and the positive charge of $\mathrm{D}$-alanine residues repels positively charged molecules such as defensins $[17,18]$. A greater net positive surface charge, as also mediated by $\operatorname{mpr} F$, would theoretically reduce the overall access of calcium-decorated DAP to its membrane target [17]. dltABCD also indirectly regulates the activity of the autolytic system, since a decrease of the cell-wall positive charge accelerates autolysin activity [18].

The cardiolipin synthase 2 gene cls2-responsible for the synthesis of cardiolipin, a negatively charged phospholipid that makes up $30 \%$ of the $S$. aureus cell membrane [19]-also plays a role in osmotic stability as a proton reservoir.

The $S$. aureus major autolysis gene atl and the lytic transglycosylase sceD (SAV 2095 sceD-like gene) play a key role in controlling cell-wall expansion, remodelling and daughter cell separation, but primarily participate in peptidoglycan turnover [17].

GraRS, a two-component regulatory system, controls the expression of several genes, including some involved in cell-wall synthesis or global regulation [17].

Several studies have investigated changes in the presence of mutations and expression of the above genetic loci in clinical and/or laboratory-derived DAP-R strains, but which molecular mechanism is a priority in the reduced susceptibility is still not well understood $[14,16,20]$.

To address all of the above mentioned issues on the possible mechanism of resistance and their interconnections, a sample of three series of MRSA clinical isogenic isolates obtained after antimicrobial exposure to TEC and DAP were included in this study. Different approaches, i.e. sequencing to detect single nucleotide polymorphisms (SNPs) in the target genes and real-time quantitative PCR (qPCR) to analyse their expression levels, were used.

This work aims to demonstrate that dltA overexpression represents a strain-independent keystone of DAP resistance (i.e. common to all DAP-R strains). This ditA overexpression was found in more than one set of DAP-R MRSA clinical isolates (in all our three sets) from different patients, in diverse geographical areas and with different genomic backgrounds. This trait, as previously reported $[14,15]$, confers an increase in D-alanylation of teichoic acids responsible for a drug electrostatic repulsion mechanism and a reduction in autolysis. Furthermore, this study showed a connection between mprF overexpression and the presence of specific point mutations in the same gene. All these hallmarks may co-operate to affect both DAP and/or glycopeptide reduced susceptibility.

\section{Materials and methods}

\subsection{Strains and molecular characterisation}

The three series of DAP-susceptible (DAP-S) and DAP heteroresistant (hDAP)/DAP-R MRSA, isolated from different patients, were epidemiologically unrelated clinical isolates from skin and soft tissue (sets 1 and 3 ) and bloodstream infections (set 2), collected from three different Italian hospitals (set 1 from 'Ospedale Civico di Cristina Benfratelli Palermo', set 2 from 'Santa Maria della Misericordia di Udine' and set 3 from 'Ospedali Riuniti di Bergamo'). Each series included an initial pre-DAP therapy strain (1A, 2A and 3A) and its isogenic isolates after development of DAP resistance during DAP administration (1B, 1C; 2B, 2C, 2D; and 3B, 3C). To verify the isogenicity of strains within each series, isolates were evaluated by the following genotypic assays: pulsed-field gel electrophoresis (PFGE); agr typing; multilocus sequence typing (MLST); and staphylococcal cassette chromosome mec (SCCmec) typing [21].

\subsection{Minimum inhibitory concentrations, macro Etest (MET) and population analysis profile-area under the curve (PAP-AUC) analysis}

MIC and MET determination for glycopeptides and DAP were conducted according to CLSI guidelines [11]. The MET procedure was performed following a protocol previously published and then evaluated for growth following the manufacturer's instructions (EAS003; AB BIODISK, Solna, Sweden) [22]. Mu3 [heterogeneous VAN-intermediate $S$. aureus (hVISA)], Mu50 [VAN-intermediate $S$. aureus (VISA)] and ATCC 29213 [VAN-susceptible S. aureus (VSSA)] were used as control strains. DAP categorisation was defined according to the EUCAST guidelines [10].

The PAP/AUC procedure was performed as previously described [23]. Colonies were counted and the $\log \mathrm{CFU} / \mathrm{mL}$ was plotted against the VAN concentration using GraphPad Prism (GraphPad Software Inc., La Jolla, CA). The ratio of AUC of the tested isolates to the AUC of $S$. aureus $\mathrm{Mu} 3$ was calculated and was then interpreted as previously described [23]. Mu3 (hVISA), Mu50 (VISA) and ATCC 29213 (VSSA) were used as control strains. In this study, strains were categorised as heterogeneous glycopeptide-intermediate $S$. aureus (hGISA) (i.e. heteroresistant to both VAN and TEC), heterogeneous TEC-intermediate $S$. aureus (hTISA) if the strain exhibited a PAP of heteroresistance versus TEC alone, hDAP (heteroresistant to DAP), quasi VISA (qVISA) if the strain showed a PAP of VAN and TEC as Mu50 but with a VAN MIC of $<8 \mathrm{mg} / \mathrm{L}$, and DAP-R if the strains had a DAP of MIC $\geq 1 \mathrm{mg} / \mathrm{L}$.

\subsection{Screening of $\delta$-haemolysin activity on $5 \%$ sheep blood agar plates}

agr operon functionality was measured by $\delta$-haemolysin production testing the strain by cross-streaking perpendicularly to $S$. aureus RN4220 as previously described [24].

\subsection{RNA extraction, retrotranscription and real-time quantitative $P C R$}

An aliquot of an overnight culture was diluted 1:50 and bacterial cells were grown in brain-heart infusion to exponential phase (optical density at $600 \mathrm{~nm}=0.4$ at $3 \mathrm{~h}$ ). RNA was then extracted, treated and quantified [16]. mRNA of the studied target genes, i.e. dltA, $m p r F, c l s 2$, atl and $s c e D$, was retrotranscribed as previously described [16].

Real-time qPCR was conducted as previously published [16]. Primers for quantification were selected to amplify a fragment of $<300 \mathrm{bp}$. gyrB was used as a normaliser (internal control) as previously published. dltA, mprF, atl and $s c e D$ real-time primers were as previously published [16], whereas the cls2 primers, amplifying a fragment of $225 \mathrm{bp}$, were $\mathrm{CV}_{158}$ 5'ATTAGAGTTAATCGTTGATGAGCAAT3' and $\mathrm{CV}_{159}$ 5'TTACGGATGTCTTGTATTAGGTCAT3'. Expression of the studied genes is represented as the increment/decrement (fold changes) 
Table 1

Phenotypic characteristics of the strains included in the study.

\begin{tabular}{|c|c|c|c|c|c|c|c|c|c|c|}
\hline \multirow[t]{2}{*}{ Strain } & \multirow[t]{2}{*}{ OXA susceptibility } & \multirow[t]{2}{*}{ OXA MIC (mg/L) } & \multirow[t]{2}{*}{ OXA MET (mg/L) } & \multicolumn{3}{|c|}{$\mathrm{MIC}(\mathrm{mg} / \mathrm{L})$} & \multicolumn{2}{|c|}{ MET (mg/L) } & \multicolumn{2}{|c|}{ PAP/AUC } \\
\hline & & & & VAN & TEC & DAP & VAN & TEC & VAN & DAP \\
\hline $1 \mathrm{~A}$ & $\mathrm{R}$ & 32 & 256 & 1 & $\leq 0.25$ & $\leq 0.25$ & 4 & 4 & VSSA & DAP-S \\
\hline 1B & $\mathrm{R}$ & 64 & 128 & 2 & $\leq 0.25$ & $\leq 0.25$ & 16 & 6 & VSSA & DAP-S \\
\hline $1 C$ & $\mathrm{R}$ & 64 & 256 & 2 & 2 & 4 & 16 & 4 & hGISA & DAP-R \\
\hline $2 \mathrm{~A}$ & $\mathrm{R}$ & 256 & 256 & 1 & 2 & 0.5 & 16 & 6 & hTISA & DAP-S \\
\hline $2 B$ & $\mathrm{R}$ & 256 & 192 & 1 & 1 & $\leq 0.25$ & 12 & 4 & hTISA & hDAP \\
\hline $2 \mathrm{C}$ & $\mathrm{R}$ & 256 & 128 & 2 & 2 & 2 & 12 & 6 & hTISA & DAP-R \\
\hline $2 \mathrm{D}$ & $\mathrm{R}$ & 512 & $>256$ & 1 & 0.5 & 0.5 & 12 & 4 & hGISA & hDAP \\
\hline $3 \mathrm{~A}$ & $\mathrm{R}$ & 2 & 4 & 1 & 16 & 0.5 & 6 & $>32$ & hGISA & hDAP \\
\hline $3 B$ & $\mathrm{R}$ & 16 & 32 & 2 & 2 & 4 & 8 & 16 & qVISA & DAP-R \\
\hline $3 C$ & $\mathrm{R}$ & 4 & 8 & 2 & 1 & 2 & 12 & 12 & qVISA & DAP-R \\
\hline
\end{tabular}

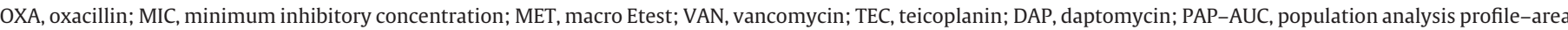

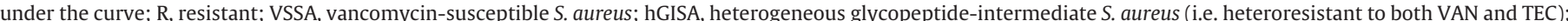

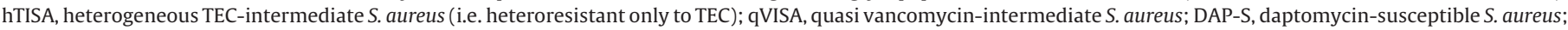
DAP-R, daptomycin-resistant S. aureus; hDAP, heteroresistant daptomycin S. aureus.

of each strain versus the daptomycin-susceptible $S$. aureus isolate within each set of isolates. For each analysis, three to five distinct biological replicates were carried out. Statistical expression analyses were performed using the Relative Expression Software Tool (REST) 2009 [16,25].

\subsection{Sequencing and sequence analysis}

Sequencing of graR, walK, mprF and cls2 genes in all strains was performed using primers designed on GenBank-deposited sequences. All amplification products were purified using a QIAquick PCR Gel Extraction Kit (QIAGEN, Valencia, CA) and were sequenced with a DNA 4000L sequencer (LI-COR, Lincoln, NE). The DNA sequence was analysed by the gapped BLAST software.

\section{Results}

\subsection{Minimum inhibitory concentrations, macro Etest and} population analysis profile-area under the curve analysis

The three sets of isogenic strains were MRSA as defined by oxacillin MICs. Set 1 was composed of two DAP-S/VSSA strains (1A and $1 B$ ) and one DAP-R/hGISA (1C), whereas set 2 included one DAP-S/hTISA (2A), one hDAP/hTISA (2B), one DAP-R/hTISA (2C) and one hDAP/hGISA (2D). Set 3 was composed of one hDAP/hGISA (3A) and two DAP-R/qVISA (3B and 3C) (Table 1 ).

\subsection{Molecular characterisation}

All set 1 strains were ST398, SCCmec IVa and had an identical ApaI/PFGE profile $(\alpha 1)$. Set 2 was ST5, SCCmec II and showed a SmaI/PFGE profile of USA100. Set 3 was ST8, SCCmec IV and belonged to the three subtypes of the same SmaI/PFGE profile, G (G1, G2 and G3) (Table 2).

\section{3. agr group and $\delta$-haemolysin}

The results showed that the set 1 ( $A, B$ and $C$ ) and set 3 (A, B and C) strains were agr type I, whereas the set 2 (A, B, C and D) strains were agr type II. All strains were negative for $\delta$-haemolysin production, indicating their proclivity to develop reduced susceptibility to DAP and glycopeptides, and confirming their glycopeptide heteroresistant phenotype, as confirmed by PAP analysis (Table 2).

\subsection{Mutations and expression studies}

Sequencing of graR, walK, $m p r F$ and $c l s 2$ in all strains was performed. Comparing the hDAP or DAP-R strains with their parental strains, it was found that graR, a regulator of genes involved in cellwall charge, did not present the point mutation Asn197 $\rightarrow$ Ser in all strains. walK $(y y c G)$ sequencing also revealed the absence of the truncating mutation of three nucleotides (CAA) from position 1111 to 1113 in all tested strains. mprF sequencing showed the mutation giving the S295L amino acid substitution only in strain 1C and the mutation leading to the T345I amino acid substitution in strain 2C, whereas a new mprF point mutation causing the amino acid substitution L291I in hDAP isolate 2D was found. No cls2 mutations were found in any hDAP or DAP-R isolates.

Fig. 1 shows the relative quantitative expression of genes related to the cell-envelope charge ( $d l t A, m p r F$ and $c l s 2$ ) and genes involved in cell-wall turnover, growth and cell separation ( $s c e D$ and $a t l$ ), expressed as fold changes compared with the DAP-S strain in sets 1 and 2 or compared with hDAP in set 3.

With regard to the cell-envelope charge genes, the DAP-R strain of series 1 (1C) having S295L in MprF and no mutation in cls2, showed that dltA, mprF, cls2, atl and sceD were upregulated compared with the DAP-S counterpart (1A). The DAP-S/VSSA strain (1B) showed no significant differences in $\operatorname{dlt} A, m p r F$ and atl expression levels versus strain $1 \mathrm{~A}$, whereas a significant downregulation was found in $s c e D$ and $c l s 2$ transcription, but not related to DAP or VAN reduced susceptibility.

In series 2, the DAP-R strain (2C) had the T345I amino acid substitution in MprF and no mutation in cls2. This strain showed an upregulation of all genes in the study compared with the DAP-S strain (2A).

In the hDAP strains of set 2 (2B and 2D), no dltA, mprF and cls2 profiles of expression associated with a DAP-R phenotype were found. The only exception was strain 2D, having L291I in MprF, in which a high transcription level of $m p r F$ was found.

Table 2

Molecular characteristics of the strains included in the study.

\begin{tabular}{|c|c|c|c|c|c|}
\hline Strain & ST & scCmec & PFGE & agr type & $\begin{array}{l}\delta \text {-Haemolysin } \\
\text { production }\end{array}$ \\
\hline $1 \mathrm{~A}$ & 398 & IVa & ApaI/ $/ 1$ & I & - \\
\hline $1 \mathrm{~B}$ & & & ApaI/ $/ \alpha 1$ & & - \\
\hline $1 C$ & & & Apal/ $\alpha 1$ & & - \\
\hline $2 \mathrm{~A}$ & 5 & II & USA100 & II & - \\
\hline $2 B$ & & & USA100 & & - \\
\hline $2 C$ & & & USA100 & & - \\
\hline $2 \mathrm{D}$ & & & USA100 & & - \\
\hline $3 A$ & 8 & IV & G1 & I & - \\
\hline $3 B$ & & & G2 & & - \\
\hline $3 C$ & & & G3 & & - \\
\hline
\end{tabular}

ST, sequence type; SCCmec, staphylococcal cassette chromosome mec; PFGE, pulsedfield gel electrophoresis; agr, accessory gene regulator. 

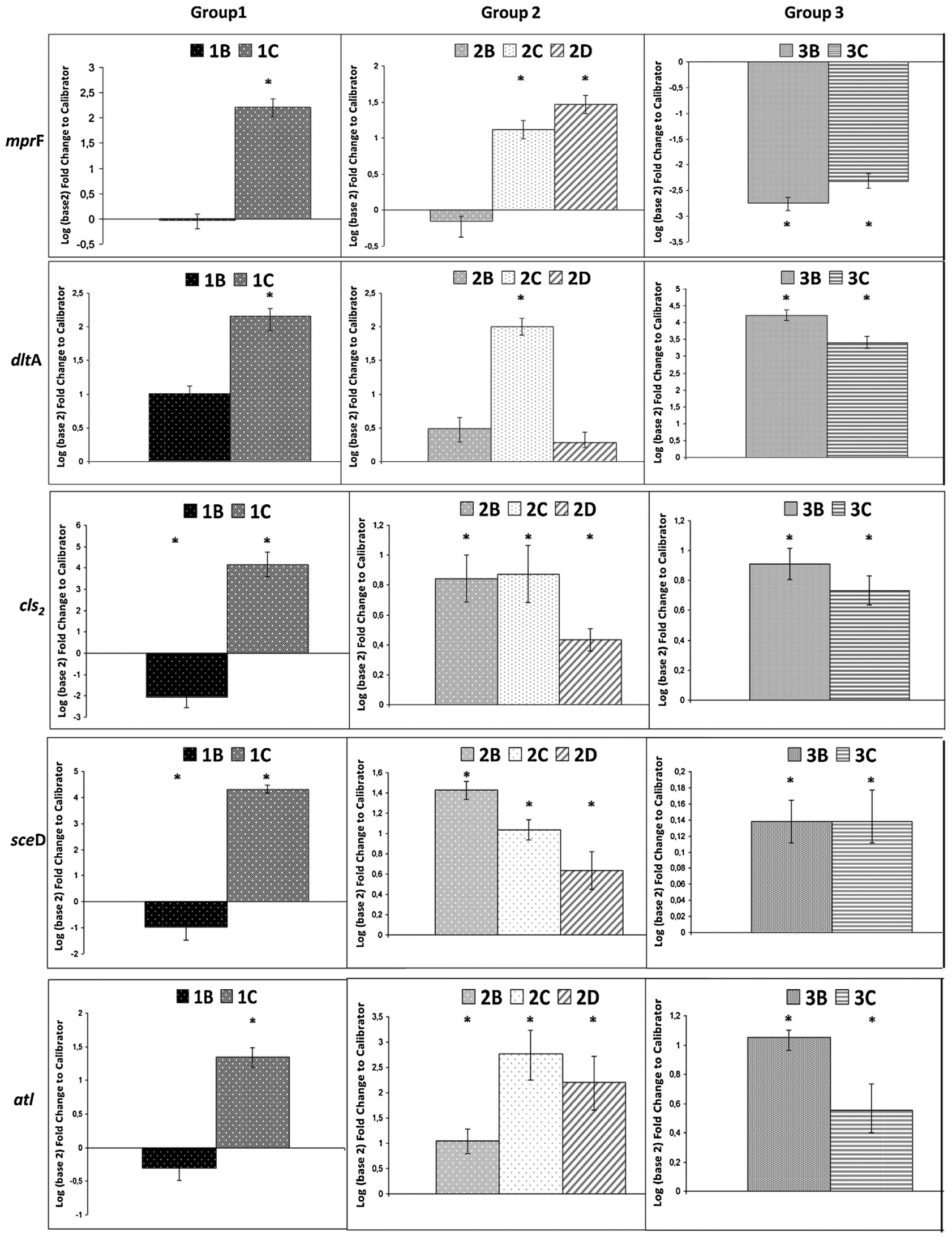

Fig. 1. Relative quantitative expression of all the studied genes in each group of strains. *Statistically significant differences $(P<0.05)$ between samples and controls. 
In contrast, the real-time qPCR data of $s c e D$ and atl evidenced an expression pattern related to their reduced susceptibility to glycopeptides phenotype, i.e. upregulation of sceD and no atl downregulation, characteristic of the hVISA phenotype (see [16]).

Series 2 isolates also included strains with an hTISA phenotype ( $2 \mathrm{~B}$ and $2 \mathrm{C}$ ). As traits in common with the DAP-R phenotype, strain 2C showed dltA and mprF upregulation, whereas strain 2D showed only mprF upregulation. As the trait in common with the hVISA phenotype, both strains exhibited sceD upregulation (see [16]).

The two DAP-R strains of series 3 (3B and $3 C$ ), having no mutations in any of the analysed genes, showed dltA, cls2, sceD and atl upregulation compared with hDAP, whereas only mprF was downregulated.

\section{Discussion}

To date, different single cases of DAP resistance development among clinical S. aureus strains under DAP therapy have been described $[2,4,26]$. Here we report our experience on three sets of DAP-S, hDAP and DAP-R MRSA isogenic clinical isolates in which we examined: (i) the development of DAP resistance and correlation with therapies; (ii) mutations and expression of the main targets involved in DAP resistance; and (iii) the co-occurrence of phenotypes of DAP resistance with glycopeptide reduced susceptibility.

Cell-wall structure modifications, depolarisation of the cell membrane and autolysis are not present at the same time in all DAP-R strains, confirming that a DAP non-susceptibility mechanism is multifactorial and is the result of multiple steps not easily detectable until full expression occurs. Identification of which molecular mechanism is the most important and/or appeared first has not yet been fully clarified.

A number of interesting findings emerged from this study. (i) The evaluation of DAP resistance development under therapy. The first consideration was regarding the development of resistance to DAP under TEC and/or DAP exposure as therapeutic regimens. In all sets, the phenotype of reduced susceptibility to both families of drugs emerged after consecutive administration of TEC and then DAP; in particular, TEC exposure induced only the hTISA/hGISA phenotype, whereas DAP treatment led to hGISA/qVISA and hDAP/DAP-R strains. This timing of antimicrobial administration favours a possible co-evolution of DAP resistance with the GISA phenotype, in agreement with other findings [14]. It is interesting to note that in only one patient (case 1) was DAP used at the suggested dosage $(6-8 \mathrm{mg} / \mathrm{kg} / \mathrm{day})$. In the other two cases, the TEC trough level was adequate for clinical efficacy [27], but DAP was underdosed in both cases.

All DAP-R isolates, but also DAP-S isolates, were $\delta$-haemolysin negative, indicating that alterations in $\delta$-haemolysin activity predispose the micro-organism to different factors such as reduced susceptibility to glycopeptides and DAP [16,24], reduced response to VAN treatment [28], reduced susceptibility to host defence cationic peptides [9] and increased biofilm formation [29].

(ii) Mutation and expression of main targets. Looking at the molecular basis of DAP resistance, this study focused on the analysis of genes related to the cell-envelope charge, cell-wall turnover, and cell growth and separation, as well as their regulation. On the basis of our recent findings and from other published studies [14], involvement of the dlt operon and $m p r F$ in DAP resistance owing to their role in the maintenance of positive staphylococcal surface charge has been highlighted. dlt operon activity has been related to increased alanylation of wall teichoic acids, resulting in a high net cell positive charge [18]. In $m p r F$, gain-in-function point mutations were identified and were associated with either excess production or increased outer cell membrane translocation of the positively charged lysyl-phosphatidylglycerol. The net result of these effects is believed to be the enhancement of relative positive surface charge [18].

On the strength of the current data, dltA overexpression is the only mechanism in common among our genotypically different series of isolates and represents a strain-independent keystone of DAP resistance in these MRSA strains. This gene, increasing the D-alanylation of teichoic acids, should confer an increased net positive charge responsible for the mechanism of resistance, essentially due to an electrostatic repulsion against $\mathrm{Ca}^{2+}$-DAP and, indirectly, due to a reduction of the autolytic system activity. These findings are in line with other prior observations performed on laboratoryinduced DAP-R MRSA strains [14] or found in meticillin-susceptible S. aureus (MSSA) clinical isolates [15].

One of the consistent features of the DAP-R strains published in the literature has been the progressive accumulation of SNPS within the mprF open reading frames. The most frequently identified are those leading to S295L [30] and T345I [20] amino acid substitutions obtained both in clinical and in vitro selected isolates $[14,20]$. Together with the hypothetical involvement of SNPs in this resistance, other authors failed to find $m p r F$ mutations [30]. In the current study, only two of four DAP-R strains, despite showing this common phenotype, possessed mutations in mprFand were overexpressed. These results are in agreement with other findings on laboratory-induced DAP-R strains [14], MSSA clinical isolates [30] and MRSA [13]. In the DAP-R strains with no mprF mutations, the resistance was only due to dltA expression. Owing to the presence of the DAP-R phenotype in strains with or without $m p r F$ mutations [16], we can hypothesise that mutations represent an adjunctive secondary trait responsible for an enhancement of mprF expression, associated with an augmentation of LP-G synthesis and/or flipping, ultimately increasing cell-envelope repulsion [18].

A different situation was found in hDAP strains where dltA was not upregulated, similarly to the hTISA and hGISA strains, reinforcing the involvement of dltA overexpression only in DAP-R strains. Moreover, a high mutation-independent transcription level of $m p r F$ was found in only one of the three hDAP strains.

The hypothetical involvement of cardiolipin synthase (cls2) mutations in DAP resistance was recently described [20]. Cardiolipin, responsible for a net negative cell-envelope charge, was not downregulated in our strains, the only situation in which this gene could be involved in the repulsion mechanism. A recent report, in which no difference in cardiolipin content was found in nine DAPS/DAP-R MRSA clinical strain pairs [31], supports our results on the non-involvement of this trait in DAP resistance.

With regard to the genes involved in cell-wall architecture, our data show that all DAP-R and hDAP strains exhibited sceD upregulation, a feature correlated with high cell-wall turnover.

(iii) DAP resistance co-occurrence with glycopeptide reduced susceptibility. All of our DAP-R strains had a glycopeptide reduced susceptibility phenotype. With the exception of dltA overexpression, characteristic of DAP-R strains, mprF, atl and sceD follow a pathway due to the co-occurrence of DAP and glycopeptide resistance, as recently published [16]. On the contrary, the hTISA phenotype showed only the previously observed hVISA profile [16] of cell-wall turnover, whereas the maintenance of cell-wall envelope charge was due to the expression profile of dltA and $m p r F$, dependent on their hDAP or DAP-R phenotype.

This investigation underlines the multifactorial nature of DAP resistance owing to a concomitance of different molecular and phenotypic events that lead to the onset of the DAP-R phenotype after antimicrobial exposure. This work analysed the molecular mechanisms of DAP resistance in three sets of clinical isogenic isolates of DAP-R MRSA with respect to the single events already published (mentioned in the text). This study could have a limitation in the relatively small sample size of DAP-R clinical isolates, but it must 
be kept in mind that DAP-R strains are still a rare phenomenon in clinical settings.

We collected two other DAP-R strains after the conclusion of this study and preliminary data appear to confirm the role of the targets already highlighted. Moreover, the major role of dltA in DAP resistance in MRSA was strengthened by data from another pair of isogenic strains, in which we found dltA overexpression only in the DAP-R parental isolate, whilst the DAP-S isolate, isolated afterwards from the same patient, acted as a 'DAP-R lacking phenotype' losing this molecular feature (data not shown). Further studies will be necessary to fully clarify the onset of this complex mechanism of resistance.

In conclusion, DAP resistance is due to a complex network of events in which different aspects can occur. A keystone can be surely represented by a mechanism of electrostatic repulsion and, indirectly, a reduction of autolysin activity due to a dltA overexpression-dependent, $c l s 2$-independent mechanism. The accumulation of adjunctive secondary factors such as the presence of mprF mutations related to increased levels of transcription may play a role in the complex phenomenon of becoming resistant.

\section{Acknowledgments}

The authors are grateful to Antony Bridgewood for the language revision of the manuscript. The authors would like to thank Dr. Dafne Bongiorno and Marianna Perez for their contribution in PAP analysis and evaluation of heteroresistance in these strains.

Funding: This study was funded by grants from the Italian Ministry of Universities [MIUR PON 01_02589].

Competing interest: None declared.

Ethical approval: Not required.

\section{References}

[1] Steenbergen JN, Alder J, Thorne GM, Tally FP. Daptomycin: a lipopeptide antibiotic for the treatment of serious Gram-positive infections. J Antimicrob Chemother 2005;55:283-8.

[2] Hayden MK, Rezai K, Hayes RA, Lolans K, Quinn JP, Weinstein RA. Development of daptomycin resistance in vivo in methicillin-resistant Staphylococcus aureus. J Clin Microbiol 2005;43:5285-7

[3] Huang YT, Hsiao $\mathrm{CH}$, Liao $\mathrm{CH}$, Lee CW, Hsueh PR. Bacteremia and infective endocarditis caused by a non-daptomycin-susceptible, vancomycin-intermediate and methicillin-resistant Staphylococcus aureus strain in Taiwan. J Clin Microbiol 2008;46:1132-6.

[4] Bayer AS, Schneider T, Sahl HG. Mechanisms of daptomycin resistance in Staphylococcus aureus: role of the cell membrane and cell wall. Ann N Y Acad Sci 2013;1277:139-58.

[5] Dvorchik BH, Brazier D, DeBruin MF, Arbeit RD. Daptomycin pharmacokinetics and safety following administration of escalating doses once daily to healthy subjects. Antimicrob Agents Chemother 2003;47:1318-23.

[6] Cui L, Tominaga E, Neoh HM, Hiramatsu K. Correlation between reduced daptomycin susceptibility and vancomycin resistance in vancomycin-intermediate Staphylococcus aureus. Antimicrob Agents Chemother 2006;50:1079-82.

[7] Sakoulas G, Alder J, Thauvin-Eliopoulos C, Moellering Jr RC, Eliopoulos GM. Induction of daptomycin heterogeneous susceptibility in Staphylococcus aureus by exposure to vancomycin. Antimicrob Agents Chemother 2006;50: 1581-5.

[8] Mangili A, Bica I, Snydman DR, Hamer DH. Daptomycin-resistant, methicillinresistant Staphylococcus aureus bacteremia. Clin Infect Dis 2005;40: 1058-60.

[9] Mishra NN, McKinnell J, Yeaman MR, Rubio M, Nast CC, Chen L, et al. In vitro cross-resistance to daptomycin and host defense cationic antimicrobial peptides in clinical methicillin-resistant Staphylococcus aureus isolates. Antimicrob Agents Chemother 2011;55:4012-18.

[10] European Committee on Antimicrobial Susceptibility Testing (EUCAST) Breakpoint tables for interpretation of MICs and zone diameters. Version
3.1, valid from 2013-02-11. EUCAST; 2013 http://www.eucast.org/fileadmin/ src/media/PDFs/EUCAST_files/Breakpoint_tables/Breakpoint_table_v_3.1.pdf [accessed 30.09.13].

[11] Clinical and Laboratory Standards Institute. Performance standards for antimicrobial susceptibility testing; twenty-third informational supplement. Document M100-S23. Wayne, PA: CLSI; 2013.

[12] Silverman JA, Oliver N, Andrew T, Li T. Resistance studies with daptomycin. Antimicrob Agents Chemother 2001;45:1799-802.

[13] Yang SJ, Nast CC, Mishra NN, Yeaman MR, Fey PD, Bayer AS. Cell wall thickening is not a universal accompaniment of the daptomycin nonsusceptibility phenotype in Staphylococcus aureus: evidence for multiple resistance mechanisms. Antimicrob Agents Chemother 2010;54:3079-85.

[14] Mishra NN, Yang SJ, Sawa A, Rubio A, Nast CC, Yeaman MR, et al. Analysis of cell membrane characteristics of in vitro-selected daptomycin-resistant strains of methicillin-resistant Staphylococcus aureus. Antimicrob Agents Chemother 2009;53:2312-18.

[15] Yang SJ, Kreiswirth BN, Sakoulas G, Yeaman MR, Xiong YQ Sawa A, et al. Enhanced expression of dltABCD is associated with the development of daptomycin nonsusceptibility in a clinical endocarditis isolate of Staphylococcus aureus. J Infect Dis 2009;200:1916-20.

[16] Cafiso V, Bertuccio T, Spina D, Purrello S, Campanile F, Di Pietro C, et al. Modulating activity of vancomycin and daptomycin on the expression of autolysis cell-wall turnover and membrane charge genes in hVISA and VISA strains. PLoS One 2012;7:e29573.

[17] Howden BP, Davies JK, Johnson PD, Stinear TP, Grayson ML. Reduced vancomycin susceptibility in Staphylococcus aureus, including vancomycinintermediate and heterogeneous vancomycin-intermediate strains: resistance mechanisms, laboratory detection, and clinical implications. Clin Microbiol Rev 2010;23:99-139.

[18] Bertsche U, Yang SJ, Kuehner D, Wanner S, Mishra NN, Roth T, et al. Increased cell wall teichoic acid production and D-alanylation are common phenotypes among daptomycin-resistant methicillin-resistant Staphylococcus aureus (MRSA) clinical isolates. PLoS One 2013;8:e67398.

[19] Short SA, White DC. Biosynthesis of cardiolipin from phosphatidylglycerol in Staphylococcus aureus. J Bacteriol 1972;109:820-6.

[20] Peleg AY, Miyakis S, Ward DV, Earl AM, Rubio A, Cameron DR, et al. Whole genome characterization of the mechanisms of daptomycin resistance in clinical and laboratory derived isolates of Staphylococcus aureus. PLoS One 2012; 7:e28316.

[21] Campanile F, Bongiorno D, Borbone S, Stefani S. Hospital-associated methicillin-resistant Staphylococcus aureus (HA-MRSA) in Italy. Ann Clin Microbiol Antimicrob 2009;8:22.

[22] Campanile F, Borbone S, Perez M, Bongiorno D, Cafiso V, Bertuccio T, et al, Heteroresistance to glycopeptides in Italian meticillin-resistant Staphylococcus aureus (MRSA) isolates. Int J Antimicrob Agents 2010;36:415-19.

[23] Wootton M, Howe RA, Hillman R, Walsh TR, Bennett PM, MacGowan AP. A modified population analysis profile (PAP) method to detect hetero-resistance to vancomycin in Staphylococcus aureus in a UK hospital. J Antimicrob Chemother 2001;47:399-403. Erratum in: J Antimicrob Chemother 2001; 48: 161.

[24] Cafiso V, Bertuccio T, Spina D, Purrello S, Blandino G, Stefani S. A novel $\delta$-hemolysis screening method to detect heteroresistant vancomycinintermediate Staphylococcus aureus and vancomycin-intermediate S. aureus. J Clin Microbiol 2012;50:1742-4.

[25] Pfaffl MW, Horgan GW, Dempfle L. Relative expression software tool (REST) for group-wise comparison and statistical analysis of relative expression results in real-time PCR. Nucleic Acids Res 2002;30:e36.

[26] Tenover FC, Sinner SW, Segal RE, Huang V, Alexandre SS, MacGowan Jr JE, et al. Characterisation of a Staphylococcus aureus strain with progressive loss of susceptibility to vancomycin and daptomycin during therapy. Int J Antimicrob Agents 2009;33:564-8.

[27] Graninger W, Wenisch C, Wiesinger E, Menschik M, Karimi J, Presterl E. Experience with outpatient intravenous teicoplanin therapy for chronic osteomyelitis. Eur J Clin Microbiol Infect Dis 1995;14:643-7.

[28] Sakoulas G, Eliopoulos GM, Moellering Jr RC, Novick RP, Venkataraman L, Wennersten C, et al. Staphylococcus aureus accessory gene regulator (agr) group II: is there a relationship to the development of intermediate-level glycopeptide resistance? J Infect Dis 2003;187:929-38.

[29] Cafiso V, Bertuccio T, Santagati M, Demelio V, Spina D, Nicoletti G, et al. agrGenotyping and transcriptional analysis of biofilm-producing Staphylococcus aureus. FEMS Immunol Med Microbiol 2007;51:220-7.

[30] Yang SJ, Xiong YQ, Dunman PM, Schrenzel J, François P, Peschel A, et al. Regulation of mprF in daptomycin-nonsusceptible Staphylococcus aureus strains. Antimicrob Agents Chemother 2009;53:2636-7.

[31] Mishra NN, Bayer AS. Correlation of cell membrane lipid profiles with daptomycin resistance in methicillin-resistant Staphylococcus aureus. Antimicrob Agents Chemother 2013;57:1082-5. 